\title{
Unbefristeter Freiheitsentzug und Lockerungen
}

\author{
Hans-Ludwig Kröber
}

Online publiziert: 17. Dezember 2009

(C) Springer-Verlag 2009

Ganz kurze Freiheitsstrafen sind umstritten, weil sie sehr kostenintensiv und von fragwürdiger Wirkung sind. Lange Freiheitsstrafen sind bei bestimmten Verbrechen unvermeidlich. Soweit sie dem Schuldausgleich und der Bewahrung des Rechtsfriedens dienen, vor allem aber dem Schutz der Allgemeinheit, wird man ihre Dauer nur sehr begrenzt vom Befinden des Verurteilten abhängig machen: so viel Strafe ist dann durch die Taten und fortbestehende Gefährlichkeit gerechtfertigt. Wo es aber weder um Sühne noch Generalprävention geht, sondern um Spezialprävention, also um die Einwirkung auf den Verurteilten, auf dass er sich künftighin als braver Bürger rechtstreu verhalte, sind die langen Strafen und insbesondere die unbegrenzten Freiheitsentziehungen ein zweischneidiges Schwert. Es zerschneidet Optionen zur fortgesetzten Kriminalität, es zerschneidet aber auch vielfach Möglichkeiten prosozialer Einbindung, die es manchmal nur in bestimmten Lebensphasen gibt und die man nicht 10 Jahre später einfach nachholen kann. Dies betrifft bestimmte Formen der Partnerschaft, der Entwicklung von Freundschaften und der Einbindung in soziale Gruppen, das Erreichen schulischer und beruflicher Abschlüsse und die Erfahrung mehr oder weniger qualifizierter Erwerbsarbeit. $\mathrm{Ob}$ beispielsweise bei einem Verlauf, in dem man einen Jugendlichen im Alter von 17 bis 27 Jahren im stationären psychiatrischen Maßregelvollzug hält, um seine Impulsivität zu kurieren, die therapeutischen Vorteile überwiegen oder der Sachverhalt, dass dem Probanden hinterher in einer entscheidenden Lebensphase 10 Jahre normaler Sozialerfahrung fehlen (Mädchen, Cliquen, Freizeit, Arbeit), ist oft schwer zu klären. Gleichermaßen stellt sich die Frage, ob nicht gerade die Belastung langgedienter Straffälliger mit

H.-L. Kröber $(\bowtie)$

Berlin, Deutschland

E-Mail: hans-ludwig.kroeber@charite.de der nun freigiebigen Anordnung von Sicherungsverwahrung jede Restchance vereitelt, dass diese Männer nun in einer Phase nachlassenden kriminellen Antriebs doch noch eine soziale Integration finden. Dümpeln sie erstmal in der Sicherungsverwahrung, wird das seit der Adoleszenz nicht bewältigte Projekt der Herstellung eines kleinen, funktionierenden, normkonformen, selbstgestalteten Lebensfeldes in Freiheit endgültig unlösbar durch den finalen Verschleiß von Selbstachtung und der Vorstellung von Eigenwirksamkeit.

Anders und deutlich gesagt: wenn es Schuldschwere und Gefährlichkeit erlauben, ist es auch aus dem wohlverstandenen Interesse der Allgemeinheit heraus ausgesprochen wünschenswert, den Aufenthalt in totalen Institutionen wie Haftanstalt oder hochgesicherter psychiatrischer Klinik möglichst schnell zu beenden, weil er - bei allem therapeutischen Bemühen - in bestimmter Weise hospitalisiert und die Fähigkeiten schwächt, die man zu einem selbstverantwortlichen Leben als Erwachsener braucht, der sich von der Frühstücksmarmelade bis zum Verwandtenbesuch um alles selbst zu kümmern hat.

Wichtig sind in diesem Zusammenhang Lockerungen, um diese - nunmehr prosoziale - Integration in das Leben in Freiheit angehen und strukturieren zu können. Es geht bei den Lockerungen in den allermeisten Fällen nicht darum, die neuen Fahrkartenautomaten bedienen zu lernen - das lernt man binnen weniger Tage. Es geht darum, sich selbst entgegen der ganz dominanten, die Vorstellungswelt dominierenden Hafterfahrung (der Haftlebensweise) nun in ein Leben in Freiheit hinein zu entwerfen, Vorstellungen davon zu entwickeln, wer man draußen sein könnte - und ob und wie man dies bewältigen kann. Gleiches gilt für die Entlassung aus dem Maßregelvollzug. Wenn dies dem Verurteilten gelingt und er den sozialen Empfangsraum auf sich zugeschnitten strukturiert hat, ist das Ziel der Lockerungen erfüllt und Entlassung angesagt. 
Lockerungen sind laut Rechtsprechung nach Maßgabe der Flucht- und Missbrauchsgefahr zu gewähren. Die Realität sieht vielfach erheblich anders aus, und Lockerungen werden verweigert, weil die Haftanstalt bzw. Aufsichtsbehörde keine Risiken eingehen will (angeprangert zu werden, falls es zu Flucht oder Rückfall kommt) und evtl. auch nicht mit der kriminalprognostischen Einschätzung von Strafvollstreckungskammer und externen Gutachtern übereinstimmt. Es mag mancherorts zudem apokryphe Gründe für Verweigerung von Lockerungen geben: Polizistenmord, frühere Fluchten/Ausbrüche, Geiselnahmen - auch wenn der Gefangene sich inzwischen gewandelt hat. Vielleicht ist das aber auch ein Gefangenen-Gerücht, das eine generalpräventive Wirkung auf solche Taten hat.
Gesicherter Grund für die vermeintlich fehlende Lockerungseignung ist Leugnen oder mangelnde Auseinandersetzung mit der Tat; zwei Beiträge in diesem Heft verdeutlichen, dass es hier aber auch keine schnellen Schlussfolgerungen geben kann. Eingehend beleuchtet wird die Langstraferproblematik - die Gewährung von Lockerungen, die Beurteilung der Voraussetzungen der gewöhnlichen wie der nachträglichen Sicherungsverwahrung wie auch die soziale Realität der Sicherungsverwahrten. Die vorliegende Bestandsaufnahme zeigt, dass noch keineswegs alles zum Besten steht, vielmehr Reformbedarf zugunsten der Verurteilten vorliegt. 